\title{
Reconfigurable antennas and link adaptation algorithms for MIMO-OFDM wireless systems
}

\author{
George D Sworo ${ }^{1 *}$, Kapil R Dandekar ${ }^{1}$ and Moshe Kam ${ }^{1,2}$
}

\begin{abstract}
In this work, we study and analyze the performance of physical layer algorithms for adaptive multiple input-multiple output orthogonal frequency-division multiplexing (MIMO-OFDM) wireless systems that employ a new class of adaptive antenna systems known as reconfigurable antennas. These antennas are capable of adaptively modifying their radiation characteristics and thus leverage pattern diversity to affect how the transmitter and receiver perceive the wireless channel. We propose a low complexity spatial adaptive modulation and coding (AMC) algorithm that uses the advantages of pattern reconfigurable antennas in concert with link adaptation to improve MIMO-OFDM link throughput. The algorithm operates in two main stages; first, it searches for the antenna configuration that yields the highest post processing signal-to-noise ratio (ppSNR) and, then, applies AMC to improve spectral efficiency. The performance of the proposed scheme is experimentally evaluated for a $2 \times 2$ MIMO-OFDM wireless system in an indoor environment.
\end{abstract}

Keywords: Link adaptation; MIMO-OFDM; Reconfigurable antennas; Adaptive modulation and coding; Spatial multiplexing

\section{Introduction}

Adaptive multiple input-multiple output (MIMO) wireless systems have been demonstrated to increase spectral efficiency and provide flexible data rates in multipath fading channels $[1,2]$. Recent research in this area has either focused on adaptive antenna systems such as pattern reconfigurable antennas [1-8] or adaptive physical layer (PHY) techniques such as link adaptation [9-13] to enhance data rates.

Several studies [2-4] have proposed pattern reconfigurable antennas for MIMO-orthogonal frequencydivision multiplexing (OFDM) systems. These antennas are capable of dynamically changing their radiation properties or patterns according to the wireless channel characteristics. Pattern reconfigurability has been shown to be effective in improving signal-to-noise ratios (SNR) at the receiver [3] and the channel capacity [4] in MIMO-OFDM systems.

Although several works $[3,4,8]$ have demonstrated the benefits of reconfigurable antennas, translating the

\footnotetext{
*Correspondence: gs342@drexel.edu

${ }^{1}$ Department of Electrical and Computer Engineering, Drexel University, 3141 Chestnut Street, Philadelphia, PA, USA

Full list of author information is available at the end of the article
}

benefits of these antennas into a practical realizable MIMO communication system is very challenging. The cost of integrating and implementing the functionality of the reconfigurable antennas into the system and their complexity of operation have inhibited the process of integration. Moreover, the use of reconfigurable antennas also introduce the need for efficient selection algorithm to leverage the radiation pattern diversity resulting from the different antenna states to improve diversity gain. Specifically, the key challenge is in the selection of the optimal radiation pattern or state from all the available states for a transceiver pair in different wireless conditions. This challenge is compounded by the large antenna state search space that grows with the number of reconfigurable antenna elements used at the transmitter and receiver. Available closed-form solutions are either computationally complex or are based on generic assumptions that may not apply to all wireless channel environments. In this work, we attempt to redress this issue by employing a low-complexity spatially adaptive scheme for joint antenna state selection and adaptive modulation and coding (AMC) for throughput enhancement in MIMOOFDM links with bit error rate constraint. We develop

\section{黑 Springer}

(c) 2015 Sworo et al. This is an Open Access article distributed under the terms of the Creative Commons Attribution License (http://creativecommons.org/licenses/by/4.0), which permits unrestricted use, distribution, and reproduction in any medium, provided the original work is properly credited. 
and test the proposed scheme in a software defined radio framework that leverages the capabilities of reconfigurable antennas and physical layer adaptation algorithms. This framework can potentially be used to integrate these antennas into practical MIMO communication systems.

Current generation standards such as 3rd Generation Partnership Project Long-Term Evolution (3GPP LTE) have employed similar techniques such as joint MIMO precoder and AMC to improve diversity gain through directional gain and interference reduction [14, 15]. Both precoding and reconfigurable-antenna-based signal processing exploit the channel state information by operating on the transmit signal using a weighting vector that essentially assigns more transmit power along beams with strong channel and vice versa. However, the key distinction between precoding techniques and antenna configuration selection algorithms is that, whereas precoding takes place at the baseband, the antenna configuration takes place at the RF domain using baseband level metrics.

The literature on AMC is vast, and for brevity, we consider the representative approaches found in [9-11]. These approaches provide the framework upon which other AMC techniques were modeled. The works in [9, 10] propose bit allocation schemes that attempt to maximize throughput over a set of modulation types given that the mean bit error rate (BER) is below a prescribed threshold. The model in [9] uses a peak BER threshold which cannot be exceeded by each subcarrier; the peak BER is adjusted iteratively until the throughput is maximized. Adaptive bit allocation is achieved by varying the signal constellation size according to the measured SNR values in each subcarrier; this allocation uses a different modulation type per subcarrier but does not consider coding. The model in [10] employs adaptive power allocation and bit loading on a per subcarrier basis, using an iterative technique known as water-filling AMC (WF-AMC).

The AMC algorithm proposed in this paper employs instead a fixed modulation type and coding rate across all subcarriers; it uses lookup tables known to both the transmitter and receiver to minimize the volume of feedback to the transmitter and reduce the computational complexity resulting from the iterative process used in $[9$, 10]. Another technique named block AMC (BL-AMC), presented in [11], builds on the model in [10] to reduce the volume of feedback information by performing AMC adaptation based only on a single OFDM symbol rather than on all subcarriers. It employs an exhaustive search algorithm for adapting a fixed modulation type and coding scheme across all subcarriers. The adaptation algorithm used in our study attempts to reduce the computational complexity by replacing the exhaustive search with fixed lookup tables.
We evaluate the experimental performance of the proposed scheme for a $2 \times 2 \mathrm{MIMO-OFDM}$ wireless system in an indoor environment. We demonstrate that the computational complexity of the proposed scheme is significantly lower than the relative values for the adaptation algorithms in $[10,11]$. Furthermore, we demonstrate the advantage of using reconfigurable antennas in synergy with link adaptation for performance enhancement in MIMO systems. The proposed algorithm provides a suboptimal but practical alternative to established algorithms that may prove difficult to implement in a realistic wireless communications system.

The paper is organized as follows: "Introduction" section outlines the related work. Section "System model" describes the system model. Section "Spatially adaptive modulation and coding" discusses the proposed algorithm for spatial adaptation in detail. Section "Experimental setup and implementation" presents the experimental setup and the implementation framework including the hardware. Section "Performance results and analysis" presents the performance results and analysis, and "Conclusions" section provides a brief summary.

\section{System model}

Figure 1 shows a high-level MIMO-OFDM model of the spatially adaptive system.

The system is constructed by concatenating a convolutional encoder with an interleaver and a symbol mapper. After the mapping, the symbols in $\mathrm{M}$-ary quadrature amplitude modulation (M-QAM) are modulated by the inverse fast Fourier transform (IFFT) and the appropriate cyclic prefix is added to reduce inter-symbol interference. The data are then split into two spatial streams that are fed into the antenna controller before transmission; the controller sets the correct antenna transmission configuration or mode. The two streams are separately transmitted from the two transmit antennas over the radio channel using spatial multiplexing technique. At the receiver, the cyclic prefix is removed and the signal is transformed back into frequency domain with an FFT prior to de-interleaving and subsequently decoded to reconstruct the received symbols.

The receiver estimates the post-processing signal-tonoise ratio (ppSNR) to be used for adaptation and then, runs the spatially adaptive modulation and coding (SAMC) control algorithm to determine the antenna configuration and transmission rates. The controller uses the lookup tables to select the indices of the configuration and AMC mode that consists of a modulation type and coding rate. It sends this indices to the transmitter via the feedback channel and transmitter uses its lookup tables to match the selected parameters.

Each of the reconfigurable antennas used in this MIMO system is able to adaptively modify its radiation 


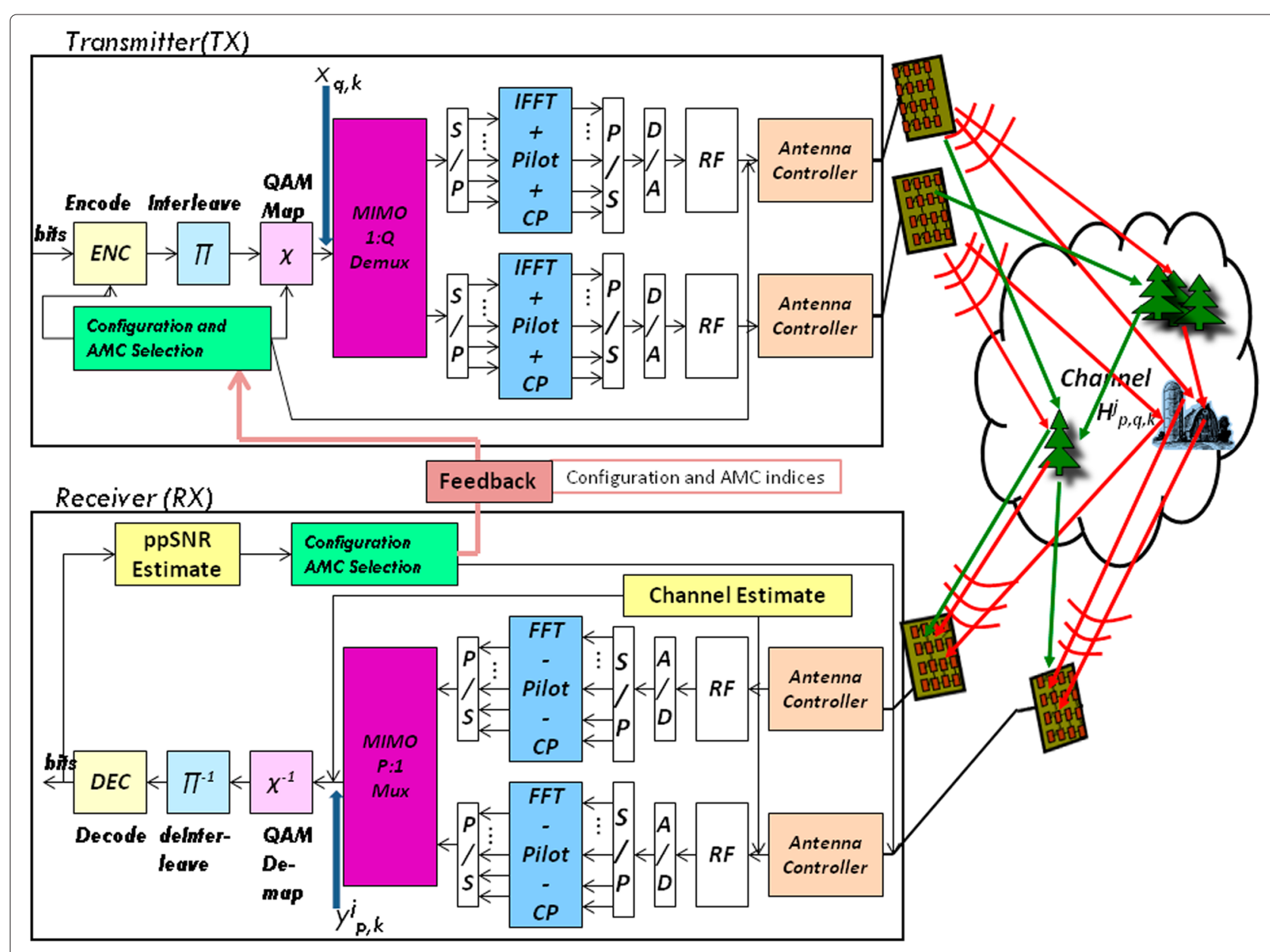

Fig. 1 System model for MIMO-OFDM using reconfigurable antennas

characteristics and thus leverage pattern diversity to impact the manner in which the transmitter and receiver perceive the wireless channel. As established in [4], "two co-located antennas with different patterns 'see' differently weighted multi-path components so that they interfere differently for the two antennas resulting in better reception". This observation motivated us to merge the benefits of antenna diversity and antenna reconfigurability to improve link capacity and SNR at the receiver [3].

We consider a MIMO-OFDM system with $Q$ transmit and $P$ receive antennas sending data across $K$ subcarriers using the $j$ th $(j=1, \ldots, J)$ antenna configuration. The OFDM sequence transmitted from the $q$ th $(q=1, \ldots, Q)$ transmit antenna on the $k$ th $(k=1, \ldots, K)$ OFDM subcarrier is represented by $x_{q, k}$. The received sequence at the $p$ th $(p=1, \ldots, P)$ receive antenna is given by

$$
y_{p, k}^{j}=\sum_{p=1}^{P} \sqrt{\frac{\varepsilon_{s}}{P}} H_{p, q, k}^{j} x_{q, k}+n_{p, k},
$$

where $y_{p, k}^{j}$ is the $P \times 1$ received vector at the $p$ th receive antenna, $H_{p, q, k}^{j}$ is the $P \times Q$ channel response matrix between the $q$ th transmit and the $p$ th receive antenna for the $k$ th subcarrier and the $j$ th antenna configuration, and $n_{p, k}$ is the $P \times 1$ additive white Gaussian noise (AWGN) at the $p$ th receive antenna for the $k$ th subcarrier. $J$ is the total number of antenna configurations, and $\varepsilon_{s}$ is the transmit energy. Expression (1) can be written as $y_{p, k}^{j}=$ $\sum_{p=1}^{P} H_{p, q, k}^{j} s_{q, k}+n_{p, k}$ where $s_{q, k}=\sqrt{\frac{\varepsilon_{s}}{P}} x_{q, k}$. After zeroforcing with successive interference cancellation (ZFSIC) equalization,

$$
\hat{s}_{q, k}=\left[H_{p, q, k}^{j}\right]^{-1} y_{p, k}^{j}=s_{q, k}+\left[H_{p, q, k}^{j}\right]^{-1} n_{p, k}
$$

where $\left[H_{p, q, k}^{j}\right]^{-1}$ is the pseudo-inverse of $H_{p, q, k}^{j}$. The ppSNR for the $p$ th stream at subcarrier $k$ and antenna configuration $j$ is defined as:

$$
\operatorname{ppSNR}_{p, k}^{j}=\frac{1}{\operatorname{var}\left(\hat{s}_{q, k}-s_{q, k}\right)}=\frac{1}{\sigma_{k}^{2}}
$$

where $\sigma_{k}^{2}$ is the noise variance of the received symbols. 
The overall ppSNR for configuration $j$ is determined as the average across transmit antennas and subcarriers.

$$
\operatorname{ppSNR}^{j}=\frac{1}{P} \sum_{p=1}^{P}\left(\frac{1}{K} \sum_{k=1}^{K} \operatorname{ppSNR}_{p, k}^{j}\right)
$$

\section{Spatially adaptive modulation and coding}

The proposed SAMC algorithm is described in Algorithm 1. It is carried out in two main stages: 1) antenna configuration selection and 2) AMC selection.

1) Antenna configuration selection: During this stage, the algorithm selects an optimal configuration $J^{*}$ that yields the highest average ppSNR. This process requires channel training and is carried out during one of the following training intervals: i) initial training interval and ii) re-training interval. The initial training interval is necessary when no prior channel training has been done. Conversely, the re-training interval prior to some initial training is only used in order to abate the effects of channel fading over time and for up-to-date channel adaptation.

i) Initial training interval: In this interval, initial channel training is carried out over all the $J$ possible configurations; ten training packets are transmitted using quadrature phase shift keying (QPSK) modulation for each of the $J$ possible configurations. After each training packet transmission, the ppSNR is calculated by taking the mean of the subcarrier ppSNR values. The average ppSNR of a specific configuration is then obtained by taking the mean of the ten transmissions. In total, 160 packets will be transmitted in this phase, and 16 average ppSNR values will be obtained. The algorithm then selects configuration $j^{*}$ that with the highest average ppSNR. We sort these 16 average ppSNR values and store the top five along with their corresponding configurations.

In this interval, there is need to transmit multiple training packets at a given configuration in order to obtain a meaningful statistic of the channel quality indicator (CQI) from post processing. However, a major challenge arises in selecting the period of the training interval: the use of a long training interval will lead to parameter adaptation based on out-dated channel characteristics; meanwhile, a short interval fails to yield a realistic statistic. Determining the optimal training period requires further analysis that deviates from the main focus of this work. Therefore, a fixed training period of ten packets was only used to obtain a CQI statistic from post processing the channel measurements. Similarly, in an attempt to minimize the re-training interval time, we selected a subset of the total configuration for the re-training phase.

ii) Re-training interval: during this interval, we re-train over the top five configurations stored in interval i); and transmit one training packet per configuration-thus, a total of five training packets. We then select the configuration that yields the highest average pPSNR out of these top five configurations.

2) AMC selection: in this stage, the algorithm selects the AMC scheme using the $\mathrm{pPSNR}^{j^{*}}$ associated with the optimal configuration in stage 1 .

The selected antenna configuration and AMC scheme are then used to transmit a scheduled number of packets. In order to minimize the loss of throughput during the training interval in stage 1 of the algorithm, we append a payload of $1 \mathrm{~KB}$ to each training packet. The size of the training packet is reduced to 32 bytes. Additionally, if the optimal configuration found during the training interval is consecutively selected, the number of packets scheduled for transmission at the optimal configuration and AMC scheme is doubled. These measures helped reduce the negative impact of training overhead on throughput gains, and not only minimized training time but provided data transmission opportunity.

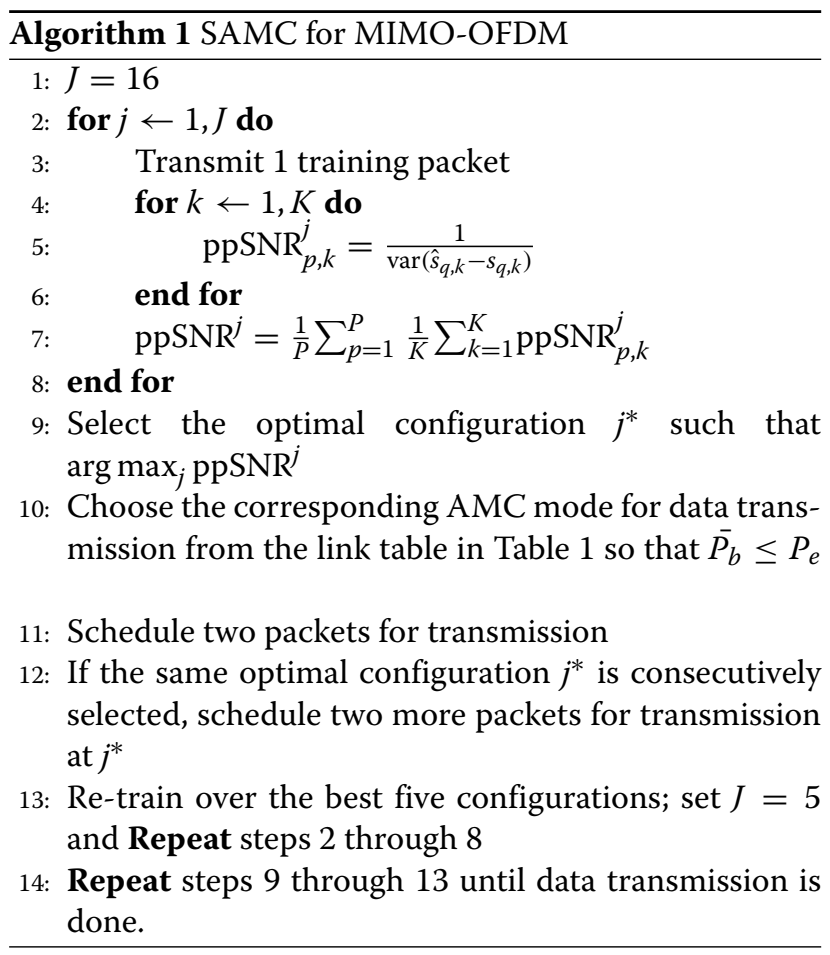

\section{AMC selection algorithms}

We propose two AMC selection algorithms: 1) highest rate selection and 2) robust rate selection. The first is a throughput maximization algorithm that selects the AMC mode with the highest data rate satisfying a target BER constraint. This AMC mode represents a fixed modulation and coding scheme across all subcarriers. The second algorithm optimizes throughput subject to constraints of 
target BER $\left(P_{e}\right)$ and target data rate $\left(\Psi_{0}\right)$. It sacrifices some throughput gain for robustness and the satisfaction of the target constraints. Robustness is achieved through optimization of the AMC mode selection. In lieu of the exhaustive search algorithms in $[10,11]$, we propose the use of predetermined link tables to obtain the best AMC in solving (6). First, we assign each of the modes to operate within a particular post processing signal-tonoise ratio (ppSNR) region $R_{\chi}$. Each region is defined by two thresholds. The thresholds are obtained by using the upper bound expression for the symbol error probability in AWGN channels [16]:

$$
\begin{aligned}
P_{\sqrt{M}} & \approx 2\left(1-\frac{1}{\sqrt{M}}\right) Q\left(\sqrt{\frac{3 m}{(M-1)} \frac{E_{b}}{N_{0}} \frac{1}{r}}\right) \\
& \leq 2 e^{-\left(\frac{3 m}{(M-1)} \frac{E_{b}}{N_{0}} \frac{1}{r}\right)}
\end{aligned}
$$

where $M$ defines the constellation size, $r$ the coding rate, $\frac{E_{b}}{N_{0}}$ is the SNR per bit, and $m=\log _{2}(M)$. The BER can be approximated by $\frac{1}{m}\left(1-\left(1-P_{\sqrt{M}}\right)^{2}\right)$.

As shown in Fig. 2, each region is delimited by the thresholds $\xi_{\chi}$ and $\xi_{\chi+1}$, such that $\xi_{\chi} \leq \operatorname{ppSNR}<\xi_{\chi+1}$. The receiver (RX) feeds back the index $\chi$ representing the AMC mode to the transmitter (TX) for adaptation. This AMC mode represents the fixed modulation and coding rate employed across all the subcarriers. When the ppSNR falls into the outage region, we keep the AMC which corresponds to region $R_{1}$, even though the target BER will not be satisfied. We restrict the set of different modulation type and coding rates to those specified in [17] (see Table 1 which shows the AMC modes and their regions of operation). Mode $\mathrm{AMC1}$, for example, uses binary phase shift keying (BPSK) and a coding rate of $1 / 2$ and operates within the region $2.2 \leq \operatorname{ppSNR}^{j^{*}}<6.8 \mathrm{~dB}$, where $\mathrm{ppSNR}^{*}$ is the post processing SNR for the selected configuration $j^{*}$.

The AMC selection procedures are explained below.

\section{Highest rate selection algorithm}

Each AMC mode will be denoted by $\chi$, where $\chi=$ $1, \ldots, \chi_{\max }$ and $\chi_{\max }$ is the total number of modes. For each $\chi$, a convolutional encoder $\varsigma_{\chi}$ with coding rates $R_{c}\left(\varsigma_{\chi}\right)$ and constellation size $M_{\chi}$, where $\log _{2} M_{\chi}=m_{\chi} \in$ $\left\{1, \ldots, m_{\chi_{\max }}\right\}$, are defined. Spectral efficiency, $\Psi$, is then obtained by solving the optimization problem $[10,11]$ :

$$
\begin{array}{ll}
\underset{\chi}{\operatorname{maximize}} & \Psi(\chi)=R_{c}\left(\varsigma_{\chi}\right) \log _{2} M_{\chi} \\
\text { subject to } & \bar{P}_{b}(\chi) \leq P_{e},
\end{array}
$$

where

$\bar{P}_{b}(\chi)=\frac{1}{P} \sum_{p=1}^{P} \frac{1}{K} \sum_{k=1}^{K} \frac{1}{m_{\chi}} Q\left(\sqrt{\frac{\left|H_{p, q, k}^{j}\right|^{2}\left|\hat{s}_{q, k}-s_{q, k}\right|^{2}}{4 \sigma_{k}^{2}}}\right)$,

and $P_{e}$ are the estimated and target bit error rates, respectively $([18,19])$.

\section{Robust rate selection algorithm}

In the design of this algorithm, we observed that the feedback information is often transmitted through a fading channel and is therefore itself prone to errors. There is a non-zero probability of feedback packet loss which may result in a mismatch of switching decisions (the transmitter may not be able to determine the correct AMC mode that the receiver sent). To decrease the occurrence of a mismatch, a change in an AMC level should only be initiated when the BER constraint cannot be achieved. Switching is minimized when the AMC mode used in the previous packet transmission is still a candidate for a subsequent transmission. The optimization problem becomes a slightly modified version of (6) with an additional constraint: $\log _{2}(1+$ ppSNR $) \geq \Psi_{0}$. This constraint ensures that the achieved data rate, $\log _{2}(1+$ ppSNR $)$, is at least equal to the target data rate, $\Psi_{0}$. The introduction of the new constraint may lead to more switching between AMC modes. However, the frequency of switching due to the additional constraint is preferable to the requirement of sending feedback after every packet transmission due to the resulting throughput savings.

The pseudocode describing the AMC selection algorithms is shown in Algorithm 2. It depicts the steps used for implementing the proposed AMC algorithms. First, the algorithm takes in the initial value of the target bit error rate $\left(P_{e}\right)$ constraint. It then computes the AMC performance thresholds as illustrated in step 3 of Algorithm 2. These thresholds are used to determine the performance regions of the lookup tables. If the objective is to maximize throughput, the "highest rate" selection algorithm is employed to choose the AMC mode. The "highest rate" selection algorithm uses Table 1, to lookup the AMC index that corresponds to the region where the measured $\mathrm{ppSNR}^{j^{*}}$ falls; thus, selecting the maximum AMC mode that satisfies the target BER constraint.

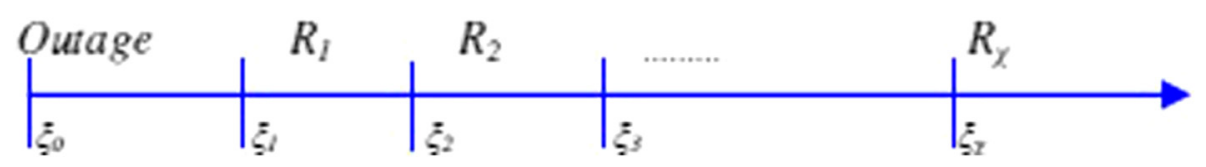

Fig. 2 Switching thresholds 
However, if robustness is preferred to throughput maximization, we utilize the "robust rate" selection algorithm to select the AMC mode. This algorithm introduces a second constraint: a target data rate that must be satisfied in addition to the target BER requirement. It first, establishes the minimum required ppSNR threshold, $\xi_{x}$, and then determines a ppSNR range over which to optimize the AMC mode selection. If the measured $\mathrm{ppSNR}$ is greater than this threshold value, the range consists of ppSNR values starting with the threshold value and incremented by an arbitrary value of $0.5 \mathrm{~dB}$ until ppSNR ${ }^{*}$. Otherwise, the range is equal to $\mathrm{ppSNR}^{j^{*}}$. Since the threshold value is the minimum requirement to satisfy both target BER and target data rate constraints, an AMC mode based on any of the ppSNR values within this range is a candidate for selection. Next, we populate 2D lookup tables for each ppSNR in the range with the AMC candidates; Table 2 shows a table for two possible target BERs and various target data rates at a ppSNR of $10 \mathrm{~dB}$. Note that an empty box in the Table 2 means that the corresponding constraints cannot be satisfied at the measured ppSNR.

As an example, suppose the target BER is $10^{-3}$, target data rate equals $1.5 \mathrm{bps} / \mathrm{Hz}$ and the measured ppSNR is $10 \mathrm{~dB}$. From Table 1, we determine that AMC3 (QPSK and coding rate $3 / 4$ ) is the minimal AMC mode that would satisfy the specified target data rate. We then calculate the minimum threshold for the target BER as in step 3 of Algorithm 2 and find that $\xi_{\chi}$ is $8.6 \mathrm{~dB}$; the ppSNR range is therefore [8.6-10] $\mathrm{dB}$. We populate four $2 \mathrm{D}$ lookup tables (one for each ppSNR value in this range) as in Table 2 for the specified target BER and target data rate constraints. And finally, select the AMC mode with the highest number of occurrence across the four lookup tables. This is the mode that minimizes the need to carry out the selection process on subsequent packet transmission.

The proposed methods significantly reduce feedback overhead since only one index representing the AMC to be used is fed back. The feedback information in our model is independent of the total number of subcarriers $(K)$ and requires only $\left\lceil\log _{2}\left(\chi_{\max }\right)\right\rceil$ compared to $K\left\lceil\log _{2}\left(m_{\chi_{\max }}\right)\right\rceil+N_{q}+\left\lceil\log _{2}\left(\chi_{\max }\right)\right\rceil$ in [10], where $N_{q}$ is the number of bits to represent the quantized power level. The price of the reduced complexity is lower accuracy - from using average channel statistics rather than the channel conditions of each subcarrier. The water-filling AMC (WF-AMC) and the block AMC (BL-AMC) are two of the alternative AMC selection algorithms with improved degree of accuracy. However, as will be demonstrated through experimental results, WF$\mathrm{AMC}$ and BL-AMC, are computationally more expensive and may prove difficult to implement in real wireless systems.

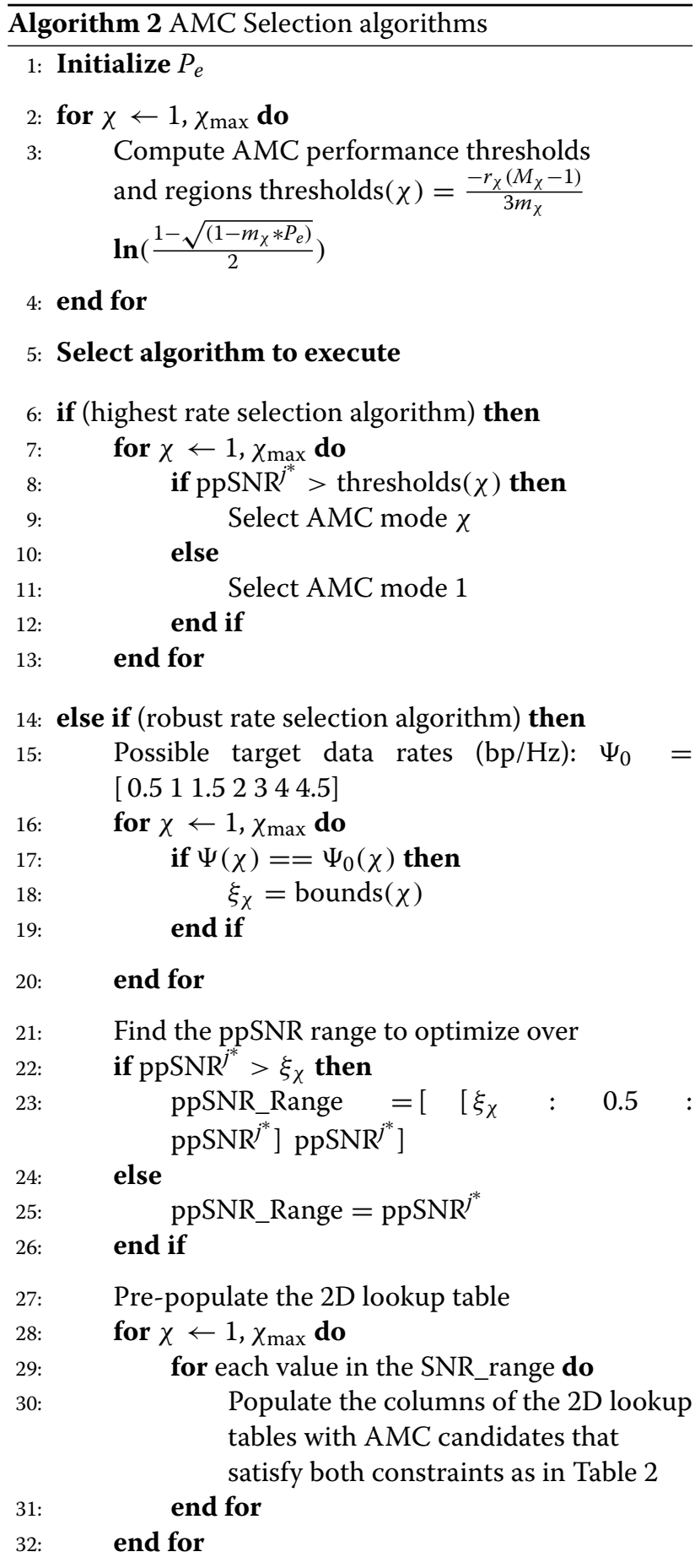

Select the AMC mode $\chi$ with the maximum occurrence across the lookup tables. This is the mode that minimizes switching

34: end if

35: Save the index of the selected AMC mode. This will be fed back to the transmitter for adaptation. 
Table 1 AMC modes and their operating regions

\begin{tabular}{|c|c|c|c|c|}
\hline AMC mode & Modulation type & Overall coding rate & Data rate $(\mathrm{bps} / \mathrm{Hz})$ & Regions for target BER $10^{-3}(\mathrm{~dB})$ \\
\hline AMC1 & BPSK & $1 / 2$ & 0.5 & $2.2 \leq \operatorname{ppSNR}^{*}<6.8$ \\
\hline AMC2 & 4-QAM & $1 / 2$ & 1 & $6.8 \leq \operatorname{ppSNR}^{*}<8.6$ \\
\hline AMC3 & 4-QAM & $3 / 4$ & 1.5 & $8.6 \leq \operatorname{ppSNR}^{*}<13.6$ \\
\hline AMC4 & 16-QAM & $1 / 2$ & 2 & $13.6 \leq \operatorname{ppSNR}^{* *}<15.3$ \\
\hline AMC5 & 16-QAM & $3 / 4$ & 3 & $15.3 \leq \operatorname{ppSNR}^{*}<21$ \\
\hline AMC6 & 64-QAM & $2 / 3$ & 4 & $21 \leq \operatorname{ppSNR}^{*}<23$ \\
\hline AMC7 & 64-QAM & $3 / 4$ & 4.5 & $\operatorname{ppSNR}^{i^{*}} \geq 23$ \\
\hline
\end{tabular}

\section{Computational complexity of SAMC}

We measure computational complexity of our algorithm by the number of elementary operations (ops) required for the algorithm to run and express it as a function of the problem size $K$ (the total number of subcarriers). For the water-filling AMC (WF-AMC) model in [10], the complexity, denoted by $T(K)$ ops, is given by

$$
T(K)=O\left(J * \varsigma_{\max }\left(2 K+2 N_{E F} * K+N_{E T} * K\right)\right),
$$

where $J$ is the total number of antenna configurations; $\varsigma_{\max }$, the number of coding rates; and $K$ is the total number of subcarriers. $N_{\mathrm{EF}}$ and $N_{\mathrm{ET}}$ denote the number of iterative searches needed in order to obtain the solutions for the "Efficientizing" and "E-tightening" subroutines in the Levin-Campello algorithm, respectively. These two parameters can grow up to $K$ in the worst case, depending on the initial bit allocation [20].

For the BL-AMC model in [11],

$$
T(K)=O\left(J * \chi_{\max } * K\right)
$$

The dependence of (8) on the number of subcarriers is due to the computation of the instantaneous Bhattacharyya factor in the bit error probability estimation. In the proposed model, the computational complexity is given by

$$
T(K)=O\left(J * K+\chi_{\max }\right) .
$$

\begin{tabular}{|c|c|c|c|c|c|c|c|}
\hline \multirow[b]{3}{*}{ BER } & \multicolumn{7}{|c|}{ Possible target rates $\Psi_{0}(\mathrm{bps} / \mathrm{Hz})$} \\
\hline & 0.5 & 1 & 1.5 & 2 & 3 & 4 & 4.5 \\
\hline & \multicolumn{7}{|c|}{ Candidate modes } \\
\hline \multirow[t]{3}{*}{$10^{-3}$} & AMC1 & AMC2 & AMC3 & & & & \\
\hline & AMC2 & AMC3 & & & & & \\
\hline & AMC3 & & & & & & \\
\hline \multirow[t]{2}{*}{$10^{-4}$} & AMC1 & $\mathrm{AMC2}$ & & & & & \\
\hline & AMC2 & & & & & & \\
\hline
\end{tabular}

Table 2 A 2D lookup table for "robust rate" selection algorithm for $\operatorname{ppSNR}=10 \mathrm{~dB}$
The two terms in (9) represent the serial selection of the optimal configuration followed by the AMC. The first term represents the complexity of selecting the optimal configuration. The second represents the AMC selection complexity, which turns out to be a constant due to the use of pre-determined lookup link tables [21].

Figure 3 illustrates the worst case scenarios of expressions (7)-(9) alongside simulations of the AMC selection algorithms. We compare, the WF-AMC [10], BL-AMC [11], the proposed SAMC algorithm, and AMC with no antenna state selection. The MATLAB function "FLOPS" [22] was used for determining the number of operations required by the selection algorithms. The results show that the computational complexity of the proposed algorithms is at least 2 orders of magnitude lower than the values for WF-AMC [10] and BL-AMC [11].

\section{Experimental setup and implementation Software defined radio (SDR) testbed}

We use the wireless open-access radio platform (WARP) designed at Rice University for protocol implementation at the PHY layer. Three main components of the WARP testbed are of interest: (a) Xilinx Virtex-II Pro FieldProgrammable Gate Array (FPGA), (b) MIMO-capable radios, and (c) 10/100 Ethernet port. The FPGA allows for MAC protocols to be written in $C$ code. The platform supports up to four radio boards which can be configured for applications similar to the $802.11 \mathrm{~g} / \mathrm{n}$ standards. Source/sink traffic and feedback of the protocols is handled over the Ethernet port [23].

\section{Reconfigurable printed dipole array (RPDA) antennas}

We use the reconfigurable printed dipole array (RPDA) [7] shown in Fig. 4 for both signal transmission and reception. These antennas have beam configurations that can be electronically controlled by adjusting the length of each dipole in the two-element array. A change in length is accomplished by biasing PIN diodes embedded in the structure of the antenna. Multiple radiation patterns are generated as a result of varying the levels of mutual coupling between array elements when the array geometry is 


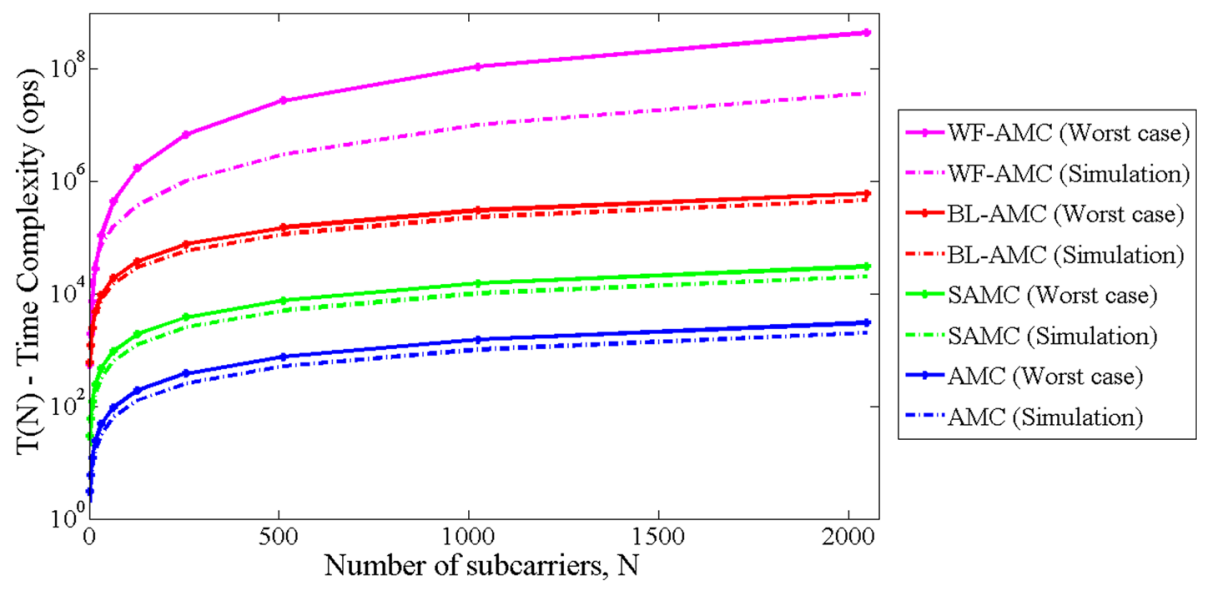

Fig. 3 Computational complexity comparison

changed [4]. We note in passing that although changing the electric length of the antenna changes the resonant frequency of the antenna, the configuration states of the array elements have been shown to resonate at a common frequency and reflection coefficient. Figure 6 in [4] illustrates a reflection coefficient of $-12.5 \mathrm{~dB}$ at a common resonant frequency of $2.484 \mathrm{GHz}$.

The RPDA antennas use PIN diode switches to achieve four different operating states. The "short" (S) configuration is used when the switches on the antenna are inactive. The active switches cause the antenna to operate in a "long" (L) configuration. One array at each end of the link therefore uses a combination of the individual antenna states to form different configurations. Table 3 shows the 16 possible combinations for the $2 \times 2$ antenna structure; Fig. 5 shows four different radiation pattern combinations for a two-antenna array system. The blue radiation pattern corresponds to antenna 1 , and the red corresponds to antenna 2 of the array. The radiation patterns on the left of Fig. 5 are generated from the short-short configurations, for antenna 1 and antenna 2, respectively. The rest are from short-long, long-short, and then long-long configurations, respectively.

To determine the pattern diversity of these antenna configurations, we evaluated the correlation coefficients based on the approach proposed by Vaughan et al. in $[24,25]$. This approach uses the radiation patterns of the antenna system and numerical integration to obtain the envelop correlation for a two-antenna system. However, it has been shown that the complex envelop correlation $\left(\rho_{c}\right)$ derived in [26] yields a more accurate result when correlation is included in the channel modeling estimation of

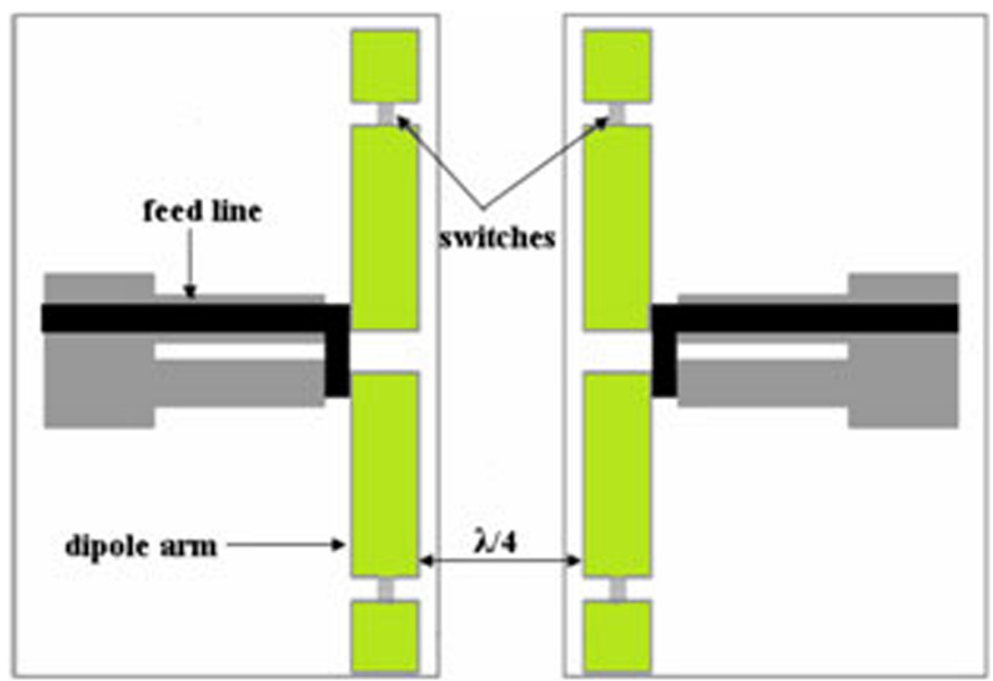

Fig. 4 Reconfigurable printed dipole array (RPDA) [7] 
Table 3 Possible configurations for $2 \times 2$ MIMO-OFDM system

\begin{tabular}{|c|c|c|c|c|c|c|c|c|c|c|c|c|c|c|c|c|c|}
\hline & & \multicolumn{16}{|c|}{ Configurations } \\
\hline & & 1 & 2 & 3 & 4 & 5 & 6 & 7 & 8 & 9 & 10 & 11 & 12 & 13 & 14 & 15 & 16 \\
\hline \multirow{2}{*}{ TX } & Ant 1 & S & S & L & S & S & $S$ & L & L & L & L & L & $\mathrm{L}$ & L & $S$ & $S$ & $S$ \\
\hline & Ant 2 & $S$ & S & L & S & L & L & L & L & L & $S$ & S & S & S & $S$ & $L$ & $\mathrm{~L}$ \\
\hline \multirow{2}{*}{$\mathrm{RX}$} & Ant 1 & $\mathrm{~S}$ & L & $\mathrm{S}$ & S & S & L & L & L & $\mathrm{s}$ & S & L & L & S & L & $\mathrm{S}$ & $\mathrm{L}$ \\
\hline & Ant 2 & $\mathrm{~S}$ & L & $S$ & L & L & L & S & L & L & $\mathrm{S}$ & S & L & L & $S$ & $S$ & S \\
\hline
\end{tabular}

capacity or BER. This expression is summarized in (10) below:

$$
\rho_{c}=\frac{\iint_{4 \pi}\left[\vec{F}_{1}(\theta, \phi) \cdot \vec{F}_{2}(\theta, \phi)\right] d \Omega}{\sqrt{\iint_{4 \pi}\left|\left[\vec{F}_{1}(\theta, \phi)\right]\right|^{2} d \Omega \iint_{4 \pi}\left|\left[\vec{F}_{2}(\theta, \phi)\right]\right|^{2} d \Omega}}
$$

where $\vec{F}_{i}(\theta, \phi)$ is the far-field radiation pattern of the antenna system when port $i$ is excited, and. is the Hermitian product (Fig. 6).

\section{Scattering environment}

The wireless environment for the experimental testing is a typical indoor office environment. The layout in Fig. 7 below shows the setting of the laboratory environment where the experiments were conducted. The office space is sectioned into cubicles by plastic materials covered in fabric and the cubicles are about $2.5 \mathrm{~m}$ tall; the walls are made of glass. The office floor is made of concrete, and the ceiling is composed of a matrix of cardboard tiles. Therefore, the scatterers and reflectors of the surrounding environment are mainly characterized by the aforementioned materials. The positions of the nodes are labeled by the numbers and the nodes are placed on desks that are 1 $\mathrm{m}$ high. Both the transmitter and receiving nodes are stationary but the channel conditions maybe influenced by sporadic human motion in between the nodes.

\section{Measurement setup}

The experimental setup used two stations. Each station is equipped with a laptop, a wireless open-access radio platform (WARP) board [23], and two reconfigurable printed dipole array (RPDA) antennas [7]. One of the stations is designated as the transmitter node and the other as the receiver node. Figure 8 shows the setup of a transmitter station. A WARP board has two radio cards, each with one antenna slot. The laptop runs the software that drives the WARP radios and the reference code for signal processing.

Prior to the experimental evaluation of our work, we conducted a test campaign to approximate the coherence time of the channel by obtaining the SNR rate profile for the fixed nodes in the measurement environment. By applying the methodology in [27], we varied the SNR across the full range of allowable received power for the WARP radio board ( -80 to $-40 \mathrm{dBm}$ ) and measured the coherence time at a speed of $0 \mathrm{mps}$. Our preliminary tests showed that the average coherence time was approximately $192 \mathrm{~ms}$. Therefore, based on this result, we infer that the channel was relatively static or slow fading and is sufficiently constant to decode the received symbols with a particular modulation rate during the coherence interval.

Our implementation uses spatial multiplexing for signal transmission between the $2 \times 2$ MIMO link established by the two stations. Specifically, we implement V-BLAST MIMO signal processing technique in concert
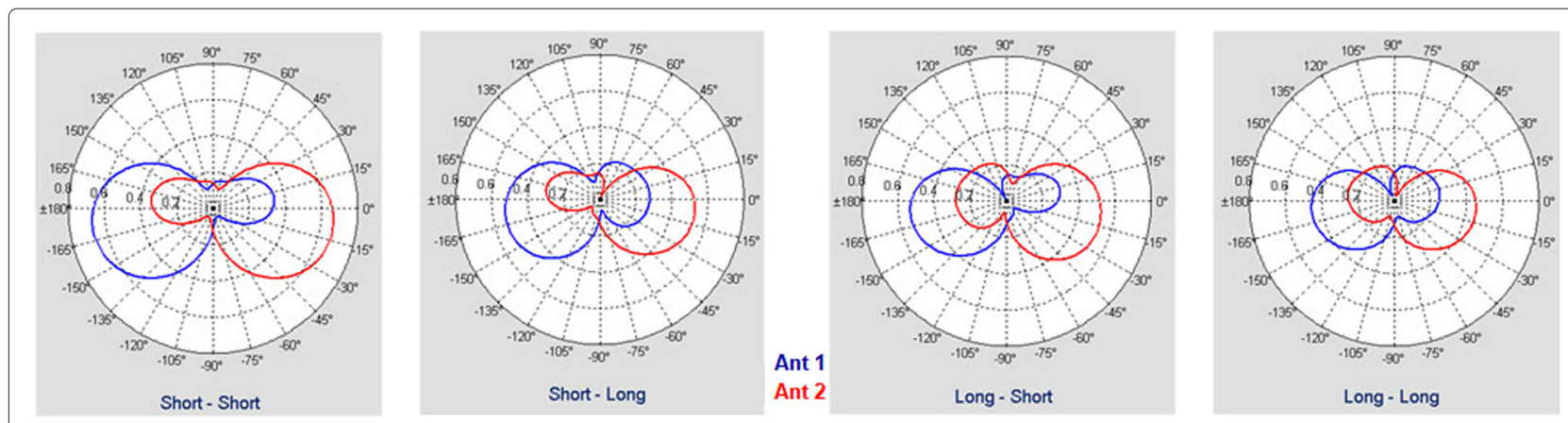

Fig. 5 Radiation patterns combinations for a two-antenna array system for all the possible antenna configurations 


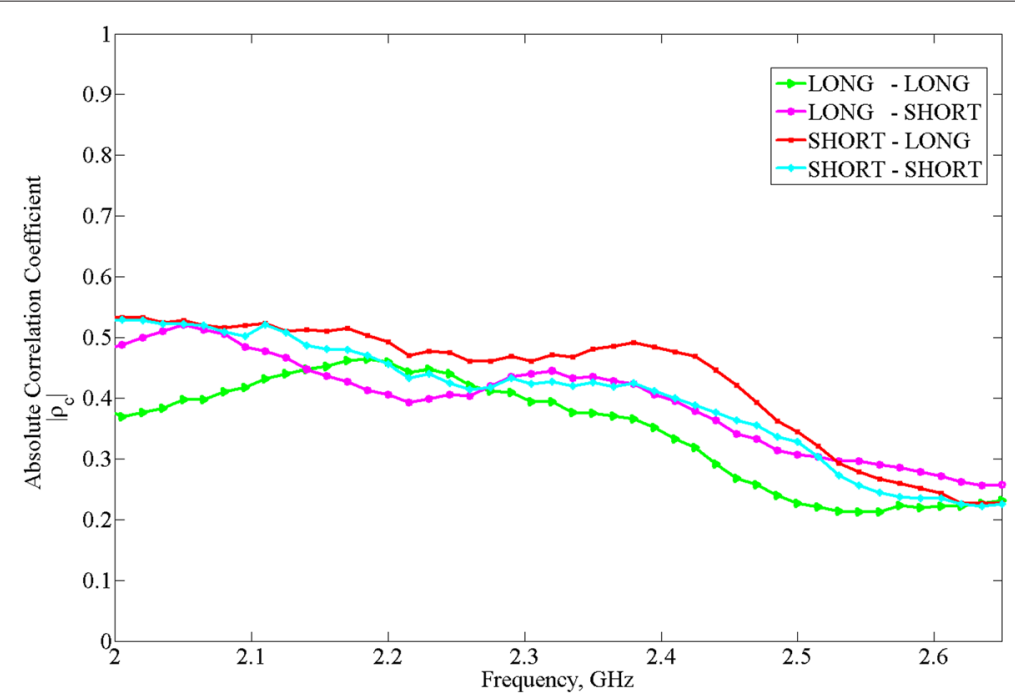

Fig. 6 The measured correlation coefficient as a function of resonant frequency for the long and short configurations in a two-element array with inter-element separation of $\lambda / 4$

with OFDM because of its ability to cope with severe channel conditions such as frequency-selective fading due to multipath. The transmission packets are based on the $802.11 \mathrm{n}$ OFDM format. The total bandwidth of $20 \mathrm{MHz}$ is divided into 64 subcarriers: 48 for data and 16 for pilot symbols and preamble. Each OFDM symbol has 80 samples (64 samples for each subcarrier plus 16 samples for cyclic prefix). Based on the manufacturer's specification, the transmission rate for the WARP radio is $10^{7}$ samples/s. To reduce noise and phase distortions, we use an over sampling factor of 4 which yields a sampling frequency of $4 \times 10^{7}$ samples/s. Based on procedure in [16], the OFDM symbol transmit time is given by (11) and the data rate by (12). And, the throughput is estimated by multiplying the result from (12) by the (1 - PER), where PER is the estimated packet error rate.

$$
\begin{aligned}
& \text { Transmit time }=\frac{\text { samples per OFDM symbol }}{\text { sampling frequency }} \\
& \text { Data Rate }=\frac{\text { bits per symbol }}{\text { transmit time }}
\end{aligned}
$$

Data are encoded using punctured convolutional codes and modulated at a carrier frequency of $2.484 \mathrm{GHz}$ using one of the four signal constellations: BPSK, QPSK, 16QAM, and 64-QAM. The convolutional encoder uses a constraint length of and code generator polynomials of 133 and 171 (in octal numbers). The puncturing matrices

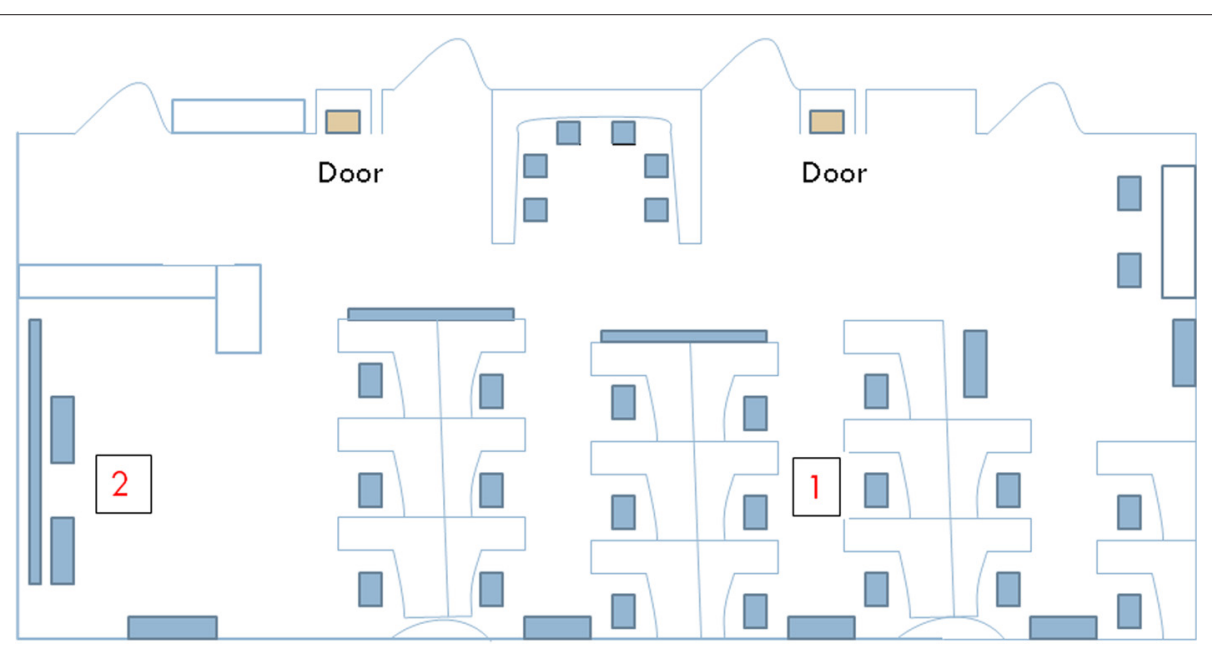

Fig. 7 The layout of the scattering environment 


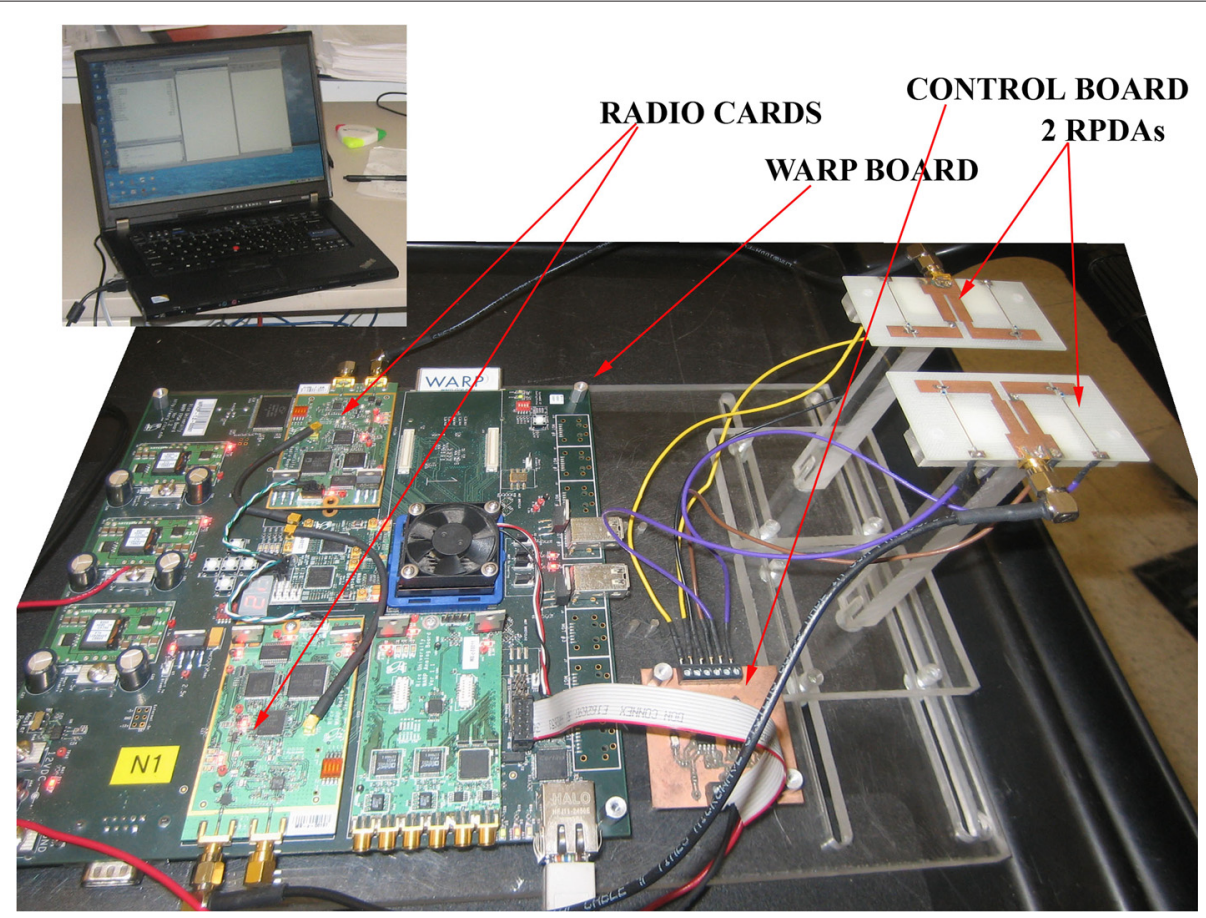

Fig. 8 An experimental communication node or station

for the relevant coding rates $(1 / 2,2 / 3,3 / 4)$ are specified in [28]. All transmissions consisted of a 24-byte header which includes a cyclic redundancy check (CRC) modulated with BPSK and bits were coded at rate $1 / 2$. The header carries a fixed channel training sequence [17] and a payload of $1 \mathrm{~KB}$ is followed by a 4-byte CRC check.

\section{Performance results and analysis}

We evaluate the experimental performance of the proposed SAMC algorithm using the measurement setup presented above. The SAMC algorithm uses the highest rate transmission AMC. We also analyze the performance of the highest rate AMC without antenna selection procedure. This is considered as the non-reconfigurable scenario where the $2 \times 2$ MIMO system is equipped with RPDA antennas that uses a fixed configuration (all antenna states are set to short configuration). We compare the performances of these two approaches with that of two other algorithms: WF-AMC [10] and BL-AMC [11].

Figure 9 illustrates the empirical ppSNR cumulative distribution functions (CDFs) of two measurement

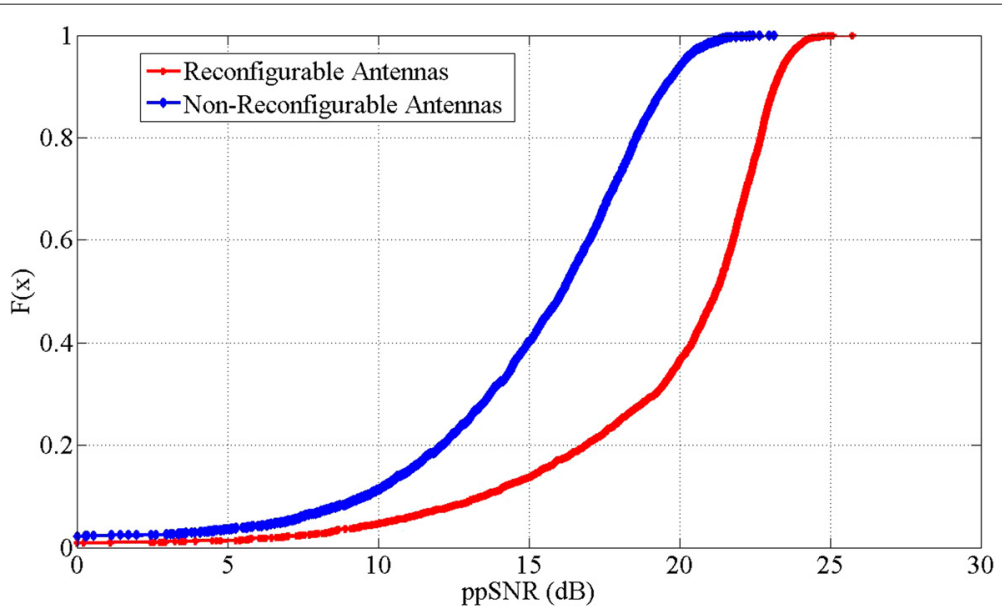

Fig. 9 Empirical ppSNR CDFs for reconfigurable AMC and non-reconfigurable AMC [29] 


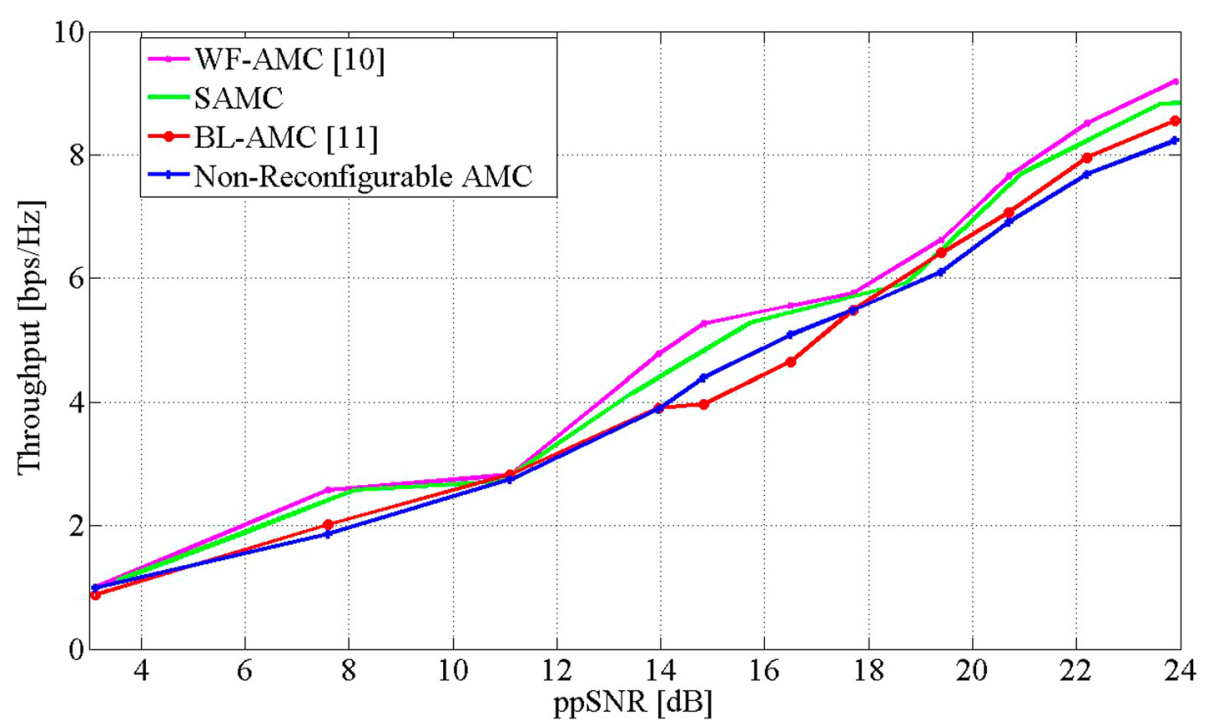

Fig. 10 Throughput comparison for $2 \times 2$ MIMO-OFDM system at $20 \mathrm{MHz}$ [29]

campaigns. In the first campaign, we used RPDA antennas for signal transmissions and measured the channel SNR. In the second, we repeated the same measurements by using non-reconfigurable (reconfigurable antenna with fixed state) antennas for signal transmissions. The curves indicate a relative gain in ppSNR of $4 \mathrm{~dB}$ when the RPDA antennas were used compared to the results from the second campaign with non-reconfigurable antennas. The performance curves indicate a gain in the reconfigurable antenna scenario due to diversity gain from antenna state switching or selection since the same set of antenna arrays were used in both scenarios.
The throughput performance curves of the WF-AMC, $\mathrm{BL}-\mathrm{AMC}$, and the proposed algorithms are shown in Fig. 10. The WF-AMC [10] model outperforms all the other schemes. This slightly better performance is due to the water-filling adaptation which is known to be optimal relative to other power allocation techniques. The proposed model, SAMC, performed better than both the spatial BL-AMC algorithm [11] and the non-reconfigurable AMC models. We note in passing that the effect of training overhead on these throughput measurements was not investigated as part of this work.

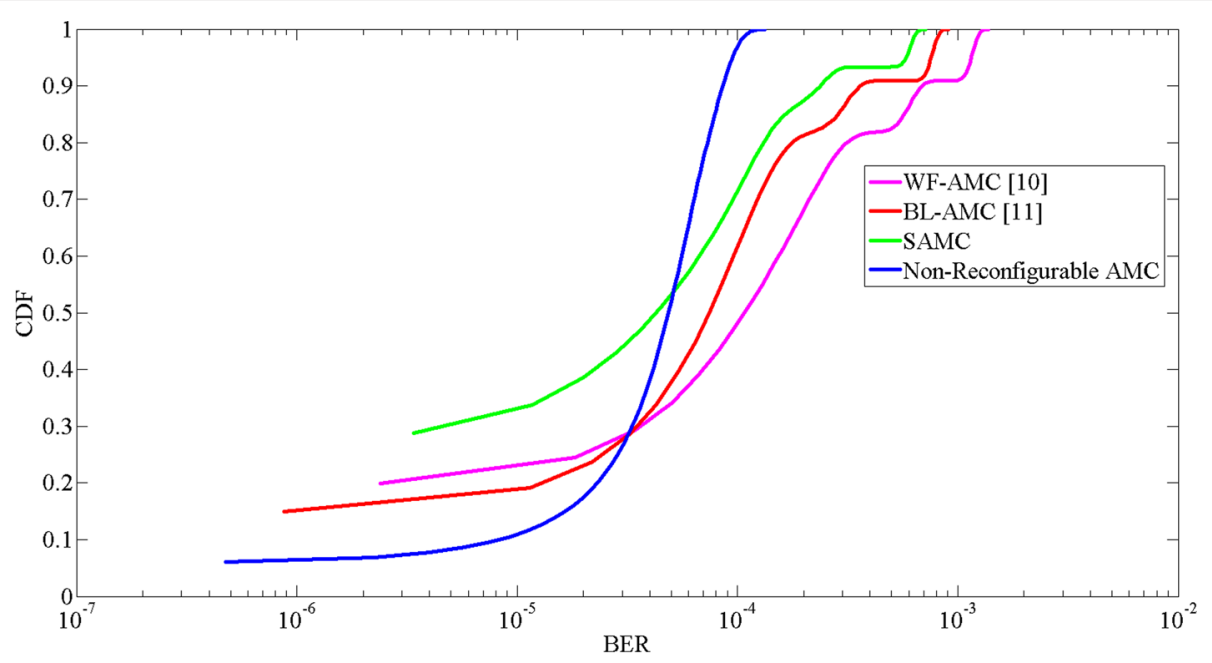

Fig. 11 BER performance comparison [29] 


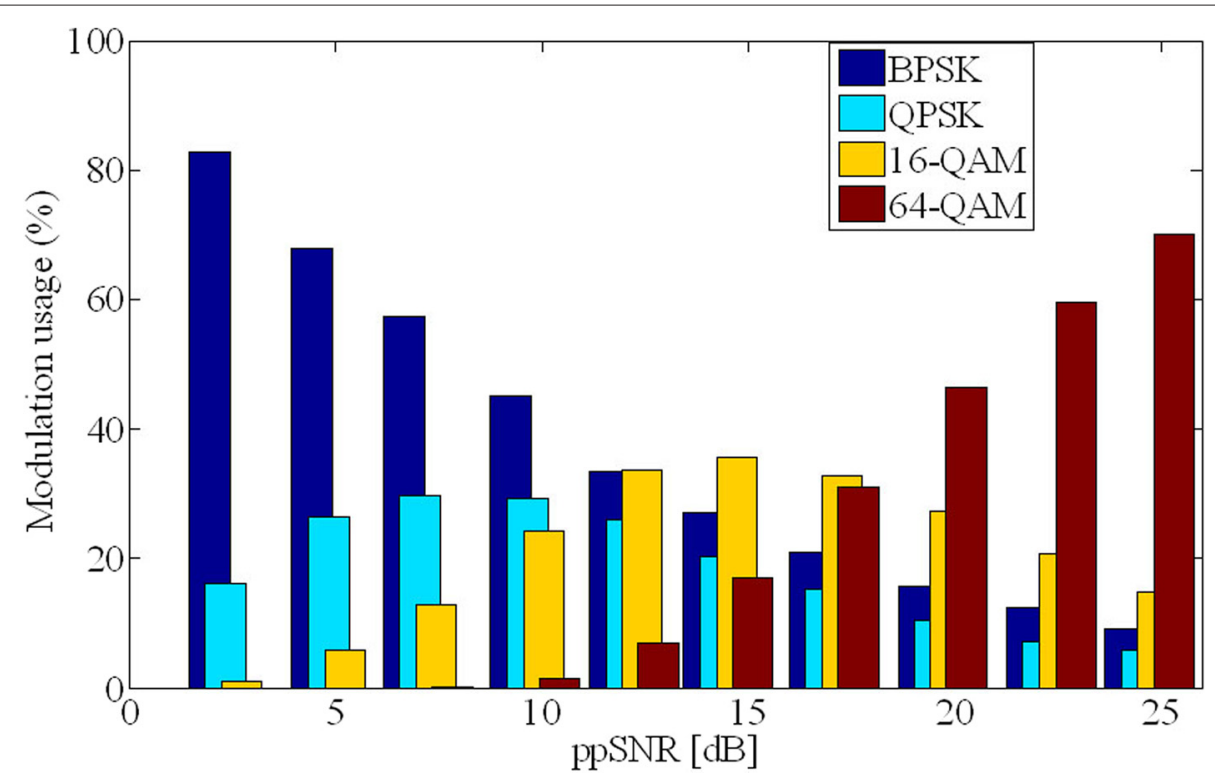

Fig. 12 The distribution of modulation use in SAMC [29]

Similarly, BER is calculated from the coarse approximation used in the optimization process based on the header information in each packet. Figure 11 plots the BER performances of the proposed models and the other models from $[10,11]$. The non-reconfigurable AMC always satisfies the target BER requirement. The other models follow similar curves but only meet the BER constraint of $10^{-3}$ at ppSNRs greater than $7 \mathrm{~dB}$. This behavior is partly due to the necessary training required for the configuration selection. Training introduces a delay which causes adaptation on outdated channel information. The random jumps above the target BER line can be explained by the use of BPSK even when the ppSNR fell in the outage region (see Fig. 2). Despite the relatively close throughput and BER performances, our approach is superior in its computational complexity performance. The computational complexity of our model is at least 2 orders of magnitude lower than the measured values for the other models in [10] and [11] (see Fig. 3).

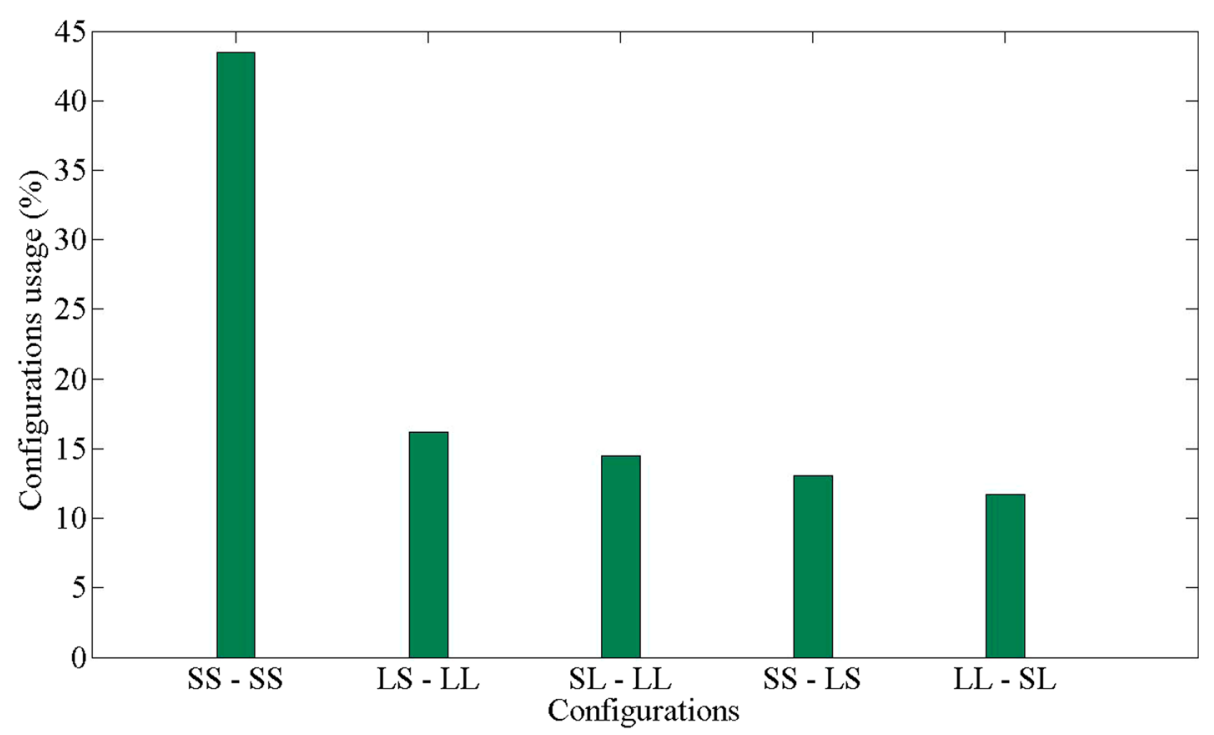

Fig. 13 Configurations usage in the proposed algorithm [29] 


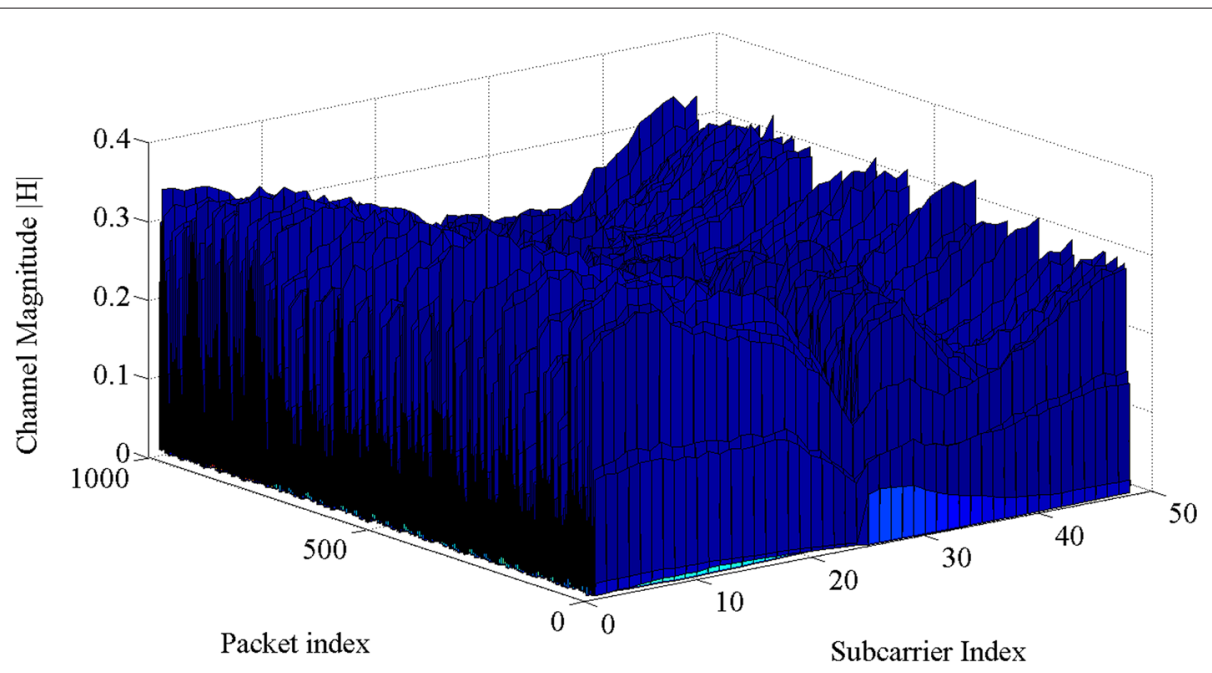

Fig. 14 Channel variation over time as measured using the active antenna configurations

In Fig. 12, we show the adaptive modulation types in terms of their fraction of usage-the percentage of time a modulation type is used during the experiment. As expected, at low ppSNR, BPSK is used most often; at moderate $(10 \mathrm{~dB})$ to middle-range ppSNR $(17 \mathrm{~dB})$, QPSK, and 16-QAM are frequently used. At high ppSNR, 64QAM dominates in usage although lower modulation types make up a combined usage of at least $30 \%$. Figure 13 illustrates the fraction of usage for the top five configurations. The horizontal axis represents the configurations used at both the transmitter and receiver. For example, SS-SS indicates that all the antennas operated in a "short" configuration. The SS-SS configuration was favored over the rest of the configurations and is chosen for more than $45 \%$ of the transmissions. The other configurations are preferred for the rest of the time due to their relatively higher PpSNR with respect to the SS-SS configuration. The benefit from switching is lower BER performance, which results in improved throughput; however, the overall throughput performance maybe suboptimal due to the use of average ppSNR for all the subcarriers. Finally, Fig. 14 illustrates the channel variation over time as measured using the active antenna configurations. It shows the flat fading nature of the wireless channel environment without deep fades.

\section{Conclusions}

A spatially adaptive modulation and coding algorithm was proposed and implemented in a software-defined radio testbed. Multiple techniques of link adaptation were employed to develop a low computational complexity, throughput enhancing model. The capabilities of reconfigurable printed dipole array (RPDA) antennas in MIMO-OFDM systems were harnessed to improve spectral efficiency. It was shown via field implementation that our model can increase post processing signal-to-noise ratio and therefore system reliability over a multipleantenna slow fading channel. Future work will investigate the effects of feedback delay on the proposed algorithm.

Competing interests

The authors declare that they have no competing interests.

\section{Acknowledgements}

The authors would like to thank Damiano Patron and Nikhil Gulati for the valuable comments and suggestions to improve the quality of the paper. The study was supported by the US National Science Foundation under grant No. 0916480

\section{Author details}

${ }^{1}$ Department of Electrical and Computer Engineering, Drexel University, 3141 Chestnut Street, Philadelphia, PA, USA. ${ }^{2}$ Department of Electrical and

Computer Engineering, New Jersey Institute of Technology, Newark, NJ, USA.

Received: 6 May 2014 Accepted: 18 May 2015

Published online: 06 June 2015

\section{References}

1. GJ Foschini, MJ Gans, On the limits of wireless communication in a fading environment when using multiple antennas. Wireless Personal Commun. 6, 131-335 (1998)

2. D Piazza, Reconfigurable antennas for adaptive MIMO communication systems. Ph.D. dissertation, Dept. Electrical and Computer Engineering. (Drexel University, Philadelphia, PA, USA, 2009)

3. A Grau, H Jafarkhani, FD Flavis, A reconfigurable multiple-input multiple-output communication system. IEEE Trans. Wireless Commun. 7(5), 1719-1733 (2008)

4. D Piazza, NJ Kirsch, A Forenza, RW Heath, KR Dandekar, Design and evaluation of a reconfigurable antenna array for MIMO systems. IEEE Trans. Antennas Propagation. 56(3), 869-881 (2008)

5. GJ Foschini, Layered space-time architecture for wireless communication in a fading environment when using multi-element antennas. Bell Lab. Technical J. 1(2), 41-99 (1996)

6. PW Wolniansky, GJ Foschini, GD Golden, RA Valenzuela, in Proceedings IEEE International Symposium on Signals, Systems, and Electronics. V-BLAST: an architecture for realizing very high data rates over the rich-scattering wireless channel (Pisa, Italy, 1998), pp. 295-300

7. D Piazza, K Dandekar, Reconfigurable antenna solution for MIMO-OFDM systems. Electron. Lett. 42(8), 446-447 (2006) 
8. J Boerman, J Bernhard, Performance study of pattern reconfigurable antennas in MIMO communications systems. IEEE Trans. Antennas Propagation. 56(1), 231-236 (2008)

9. AM Wyglinski, F Labeau, P Kabal, An efficient bit allocation algorithm for multicarrier modulation. Wireless Commun. Netw. Conf. 2, 1194-1199 (2004)

10. K Kong, A Ekbal, JM Cioffi, ST Chung, Adaptive modulation and coding (AMC) for bit-interleaved coded OFDM (BIC-OFDM). IEEE Trans. Wireless Commun. 5(7), 3197-3201 (2006)

11. CK Sung, S Chung, J Heo, I Lee, Adaptive bit-interleaved coded OFDM with reduced feedback information. IEEE Trans. Wireless Commun. 55(9), 1649-1655 (2007)

12. A Goldsmith, S Chua, Variable-rate variable-power MQAM for fading channels. IEEE Trans. Commun. 45(10), 1218-1230 (1997)

13. D Qiao, S Choi, KG Shin, Goodput analysis and link adaptation for IEEE 802.11a wireless LANs. IEEE Trans. Mobile Comput. 1(4), 278-292 (2002)

14. IF Akyildiz, DM Gutierrez-Estevez, EC Reyes, The evolution to 4G cellular systems: LTE-Advanced. Elsevier J. Phys. Commun. 3, 217-244 (2010)

15. RT Becker, Precoding and spatially multiplexed MIMO in 3GPP long-term evolution. High Freq Electron (2009). [online] Available: http://highfreqelec. summittechmedia.com/OCt09/HFE1009_Becker.pdf

16. A Goldsmith, Wireless communications. (Cambridge University Press, London, 2005)

17. IEEE 802.16 Working group IEEE standard for local and metropolitan area networks. Nov. 2004. [Online] Available: https://standards.ieee.org/ findstds/standard/802.16-2004.html

18. JM Cioffi, A multicarrier primer. Standford University. last accessed Sept. 2010. [online] Available: http://www.stanford.edu/group/cioffi/ documents/multicarrier.pdf

19. JG Proakis, Digital communiations, 4th Ed. (McGraw-Hill, New York, 2000)

20. J Cioffi, EE379C-Digital communication: signal processing. (Stanford University, Stanford, CA, 2008). [online] Available: http://www.stanford. edu/class/ee379c

21. I Parberry, Algorithm analysis and computational complexity. (University of North Texas, TX, 2001). [online] Available: http://larc.unt.edu/ian/books/ free/lnoa.pdf

22. A Quarteroni, F Saleri, Scientific computing with Matlab. (Springer, Berlin, 2003)

23. WARP Research Platform, Rice University. [Online]. Available: http://warp. rice.edu/

24. RG Vaughan, JB Antenna, Diversity in mobile communications. IEEE Trans. Vehicular Technol. 36(4), 149-172 (1987)

25. CB Dietrich, K Dietze, RJ Nealy, WI Stutzman, Spatial polarization and pattern diversity for wireless handheld terminals. IEEE Trans. Antennas Propagation. 49(9), 1271-1281 (2001)

26. P Kyritsi, DC Cox, RA Valenzuela, PW Wolniansky, Correlation analysis based on MIMO channel measurements in an indoor environment. IEEE J. Selected Areas Commun. 21(5), 713-720 (2003)

27. J Camp, E Knightly, Modulation rate adaptation in urban and vehicular environments: cross-layer implementation and experimental evaluation. IEEE/ACM Trans. Netw. 18(6), 1949-1962 (2010)

28. P Frenger, PO Tony, A Svensson, Multi-rate convolutional codes. (Chalmers University Technology, Gotenberg, Sweden, Technology Republic 21, 1998)

29. GD Sworo, M Kam, K Dandekar, in Proceedings of the 46th Annual Conference on Information Science and Systems (CISS). Performance of link adaptation algorithms and reconfigurable antennas for MIMO-OFDM wireless systems (Princeton USA, 2012)

\section{Submit your manuscript to a SpringerOpen ${ }^{\circ}$ journal and benefit from:}

- Convenient online submission

Rigorous peer review

- Immediate publication on acceptance

- Open access: articles freely available online

- High visibility within the field

- Retaining the copyright to your article

Submit your next manuscript at $\boldsymbol{\triangleright}$ springeropen.com 